\title{
Radial Velocity Variations from Starspots
}

\author{
Artie P. Hatzes \\ McDonald Observatory, University of Texas, Austin, TX, USA
}

\begin{abstract}
In this contribution the radial velocity (RV) variations expected for starspots on solar-type stars are examined. The spot-induced RV amplitude is found to vary linearly with spot filling factor and is less than $1 \mathrm{~m} \mathrm{~s}^{-1}$ for spot sizes comparable to large sunspots and as high as $20 \mathrm{~m} \mathrm{~s}^{-1}$ for spot filling factors of $1 \%$. Also, for a given spot size the RV amplitude increases linearly with $v \sin i$. All of these findings confirm the results of Saar \& Donahue (1997). It is also shown that two spectral lines with different temperature sensitivity can have different RV amplitudes which may provide a diagnostic for confirming planet detections. The RV variations due to starspots correlate well with the displacement of the line core and centroid and this can be used to correct RV measurements for the effects of cool starspots.
\end{abstract}

\section{Introduction}

Techniques capable of measuring stellar radial velocities (RV) to a precision of $10 \mathrm{~m} \mathrm{~s}^{-1}$ have already resulted in the first discoveries of Jovian-mass planets around other stars. The next breakthrough in techniques, ones that can measure stellar RV to a precision better than $1 \mathrm{~m} \mathrm{~s}^{-1}$ holds the promise of detecting the first Saturn- and Neptune-mass planets. However, at this level of precision the intrinsic variability of the star becomes a concern. In particular starspots can induce RV variations which can obscure the signal due to a planetary companion. Recently, Saar \& Donahue (1997) showed that the RV variations in stars increase with rotation and the level of activity. In this contribution the expected RV variations due to starspots are investigated using numerical simulations.

\section{Numerical Simulations}

Synthetic spectral lines were generated using a disk integration scheme with 200 $\times 200$ cells on the visible stellar disk. Local line profiles for the spotted $\left(T_{\text {spot }}\right.$ $=3700 \mathrm{~K})$ and photospheric regions $\left(T_{\text {phot }}=4900 \mathrm{~K}\right)$ were provided by Kurucz model atmospheres. The resolution of the data was $0.043 \AA$. The synthetic stellar spectrum was combined with a "fake" iodine absorption line spectrum (a dense forest of absorption lines much narrower than the stellar lines) which provided the wavelength reference for the RV calculation. Two spectral lines with different temperature sensitivities were considered: Fe I $6430 \AA$ and Ca I $6439 \AA$. 


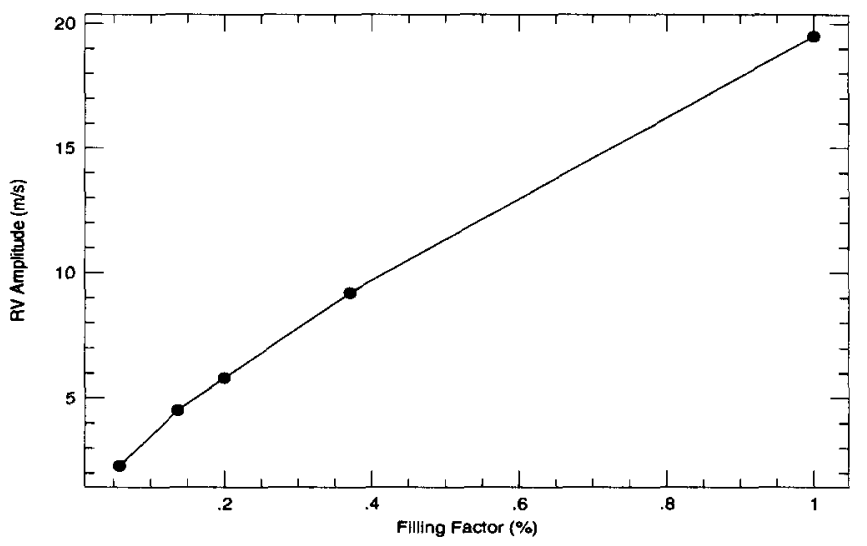

Figure 1. The RV amplitude as a function of spot filling factor.

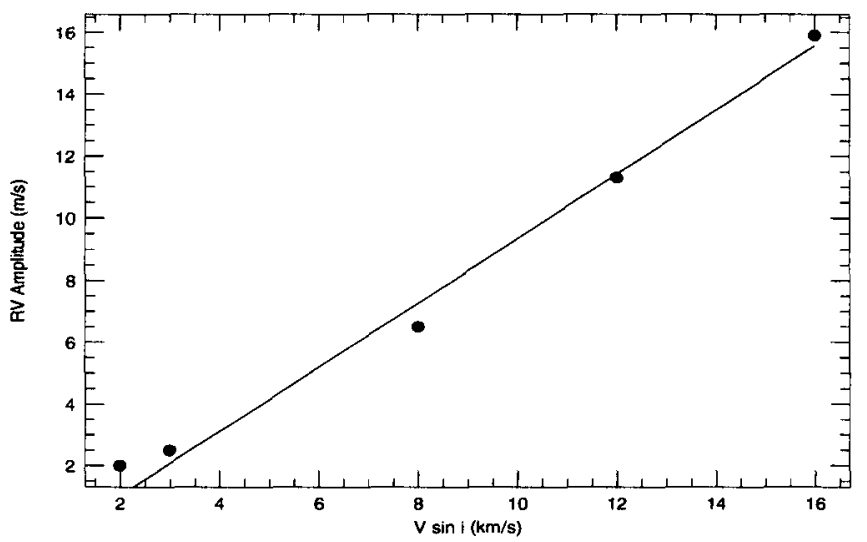

Figure 2. The RV amplitude versus $v \sin i$ for a spot of fixed size (radius $=3^{\circ}$ ) located at $10^{\circ}$ stellar latitude.

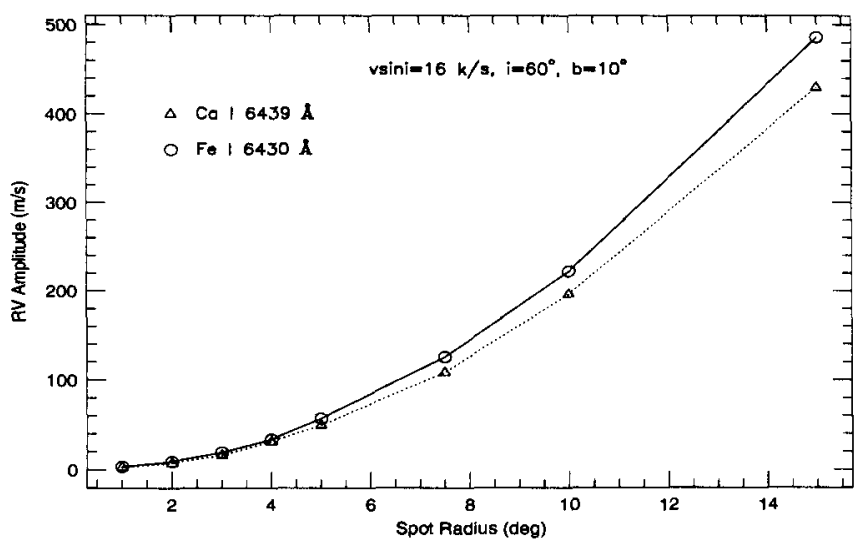

Figure 3. The RV amplitude versus spot radius for the Fe I $6430 \AA$ line (circles) and the $\mathrm{Ca} I 6439 \AA$ line (triangles). 


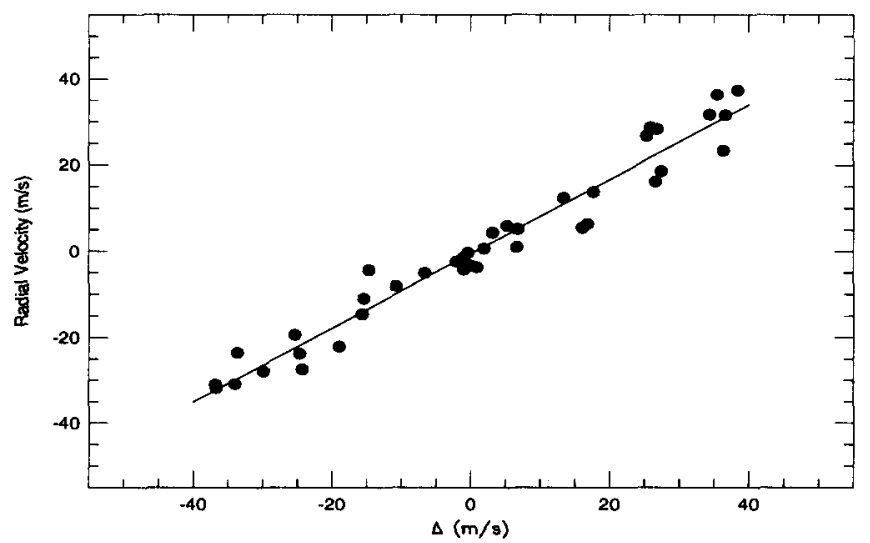

Figure 4. The RV amplitude versus the $\Delta$, the RV difference between the centroid and the core of the Fe I $6430 \AA$ line. The points represents a variety of 1 -spot models. The line represents a least squares fit.

\section{Results}

Figs. 1-3 summarize the results of the simulations. Fig. 1 shows the RV amplitude as a function of spot filling factor (in percentage) for a star with a projected rotational velocity of $3 \mathrm{~km} \mathrm{~s}^{-1}$. For a fixed spot area the amplitude of the RV variations increases linearly with $v \sin i$ as demonstrated in Fig. 2. Both these figures confirm the results of Saar \& Donahue (1997). For spotted stars the amplitude of the spectral distortions due to spots can differ between lines of different temperature sensitivity. Thus different spectral lines can show a different RV amplitude. This is demonstrated in Fig. 3, which shows the RV amplitude for a single spot of varying radius located at $10^{\circ}$ stellar latitude on a star with $v \sin i=16 \mathrm{~km} \mathrm{~s}^{-1}$. The triangles represent the RV amplitude for the Ca I $6439 \AA$ line and the circles are for the Fe I $6430 \AA$ line, a line whose strength is relatively temperature insensitive. Since the Ca I $6439 \AA$ line increases its strength with decreasing temperature the spectral distortions have a smaller amplitude and this is reflected in smaller RV variations. For stars with modest rotation rates the Ca I $6439 \AA$ line can have a spot-induced RV amplitude that is $10-15 \%$ lower than that of the Fe I $6430 \AA$ line.

\section{Correcting the RV Variations from Starspots}

The RV variations due to starspots arise from spectral distortions, and consequently these variations should be accompanied by measurable changes in the spectral line shapes. If one can calibrate the RV versus line shape changes it may be possible to correct the RV measurements for the contribution due to spots. A strong correlation was found between the RV amplitude and the velocity difference $(\Delta)$ between the core and the mid-point (at half the depth) of the spectral line. Fig. 4 shows the RV amplitude versus $\Delta$ for a wide range of single-spot models on a star with $v \sin i=3 \mathrm{~km} \mathrm{~s}^{-1}$. The line core position was determined via a parabolic fit to the bottom 3 points of the line profile. 


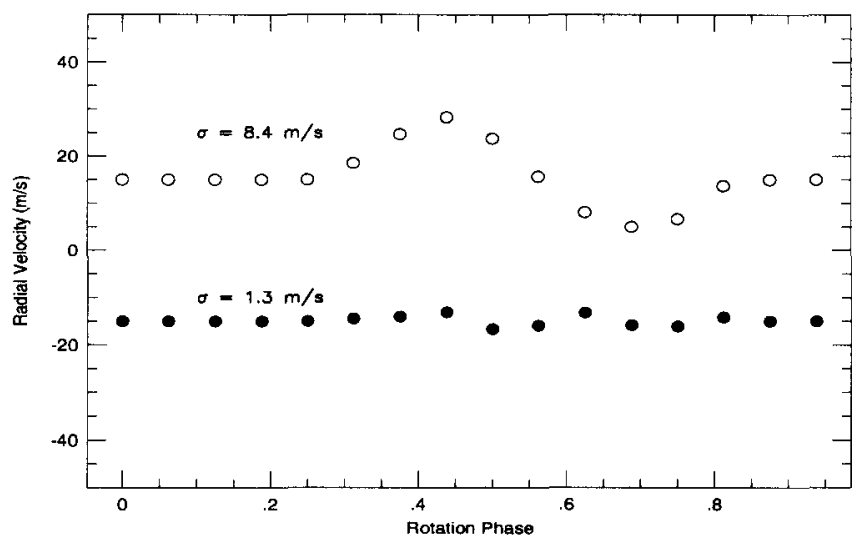

Figure 5. (Open circles) The RV variations from a random distributions of 6 spots. These were generated using the Fe I $6430 \AA$ line. (Solid points) The corrected RV values using the linear relationship in Fig. 4. The two curves have been displaced for better viewing.

The centroid was measured by taking the line bisector value at one-half the line depth. The circles in Fig. 5 show the RV variations from a random distribution of 6 spots (radius $=2^{\circ}$ ) centered near one rotation phase. The solid points represent the RV corrected variations using the least squares fit (line) of Fig. 4.

\section{Conclusions}

1. The RV variations from starspots varies linearly with $v \sin i$ and filling factor. These results confirm the initial findings of Saar \& Donahue (1997).

2. Late-type stars with modest rotation may be ill-suited for ultra-precise RV measurements due to increased levels of activity and the $v \sin i$ "amplification effect." One must either limit RV planet searches to the most inactive stars or apply an RV correction term due to starspots.

3. RV measurements made from lines of different temperature sensitivities can have different RV amplitudes. These can be used to distinguish RV variations due to starspots from those due to a planetary companion.

4. The use of line shape measurements may be used to correct for the RV variations due to spots. In particular the RV difference between the line centroid and the position of the core of the line may provide an adequate spot "correction term" for improving the RV measurement (for planet searches) by a factor of $2-10$.

Acknowledgments. This work was supported by NASA grant NAG5-4384.

\section{References}

Saar, S.H. \& Donahue, 1997, ApJ, 485, 319. 


\section{Discussion}

Isaak: In addition to sampling closely over a spectral line, one needs to sample frequently. Then one can reduce modulation effects, as we showed in Claverie et al. (Nature 1982).

Hatzes: I'll take a look at that paper.

Cameron: The amplitude of variation is likely to increase as the square of the rotation rate - you showed that the velocity residuals for a fixed spot configuration increases linearly with rotation rate, but in reality the total spot area also increases roughly with rotation rate. If you really want to calibrate this effect out reliably, you'll need to sample regularly on time scales shorter than both the rotation period and the spot lifetime.

Hatzes: This is true. The simulation was made for a fixed spot size, and the radial-velocity amplitude varied linearly with $v \sin i$. Of course, more rapidly rotating stars have more spots than slower rotators do. And I agree that this kind of calibration should be done on an active, short-period, solar-type star.

Griffin: Would not the best template of all be an independent spectrum of the same star at the relevant phase?

Hatzes: I think the best template would be several observations, preferably covering a rotation period, if that is known, co-added so as to minimize any line distortions due to spots. That is what was done for this simulation.

Kürster: What is your estimate of how easy it will be to provide reliable corrections for the radial velocity effects induced by activity? In particular, how representative are the random spot models you used in order to achieve such a correlation?

Hatzes: I really don't know. These results based on simple modelling are encouraging, but need to be investigated further. The real test would be to apply them to a short-period, fairly active star by making radial-velocity and lineprofile measurements during a rotation period and seeing how well the corrections work. 